\title{
Cultura organizacional: conceptualizaciones y metodologías detrás de un concepto complejo
}

\author{
Organizational culture:Theories and methodologies behind a complex \\ concept \\ Juliana Frassa \\ Departamento de Sociología. Facultad de Humanidades y Ciencias de la Educación. Universidad Nacional de La Plata, Argentina \\ mfrassa@ceil-piette.gov.ar
}

Fecha de recepción: 1-6-2010

Fecha de aceptación: 29-11-2010

Resumen: El abordaje de la dimensión cultural de las empresas es una temática recurrente, pero no por ello menos controvertida y compleja. Ya sea en su conceptualización, operacionalización y/o utilización desde la gestión empresarial, la cultura organizacional presenta heterogéneos tratamientos teóricos y empíricos que dificultan la construcción de un consenso entre los investigadores.

Considerando a la empresa como un lugar de creación simbólica e identitaria, dadora de significados y sentidos a la acción de sus miembros, el presente artículo tiene por objetivo revisar críticamente los principales abordajes teórico-metodológicos que, desde los Estudios de la Organización y del Trabajo, tuvo la cultura organizacional. Asimismo se propone identificar las principales tensiones y consensos presentes en torno al concepto y ponderar su utilidad heurística.

Palabras clave: cultura organizacional, escuelas teóricas, metodología.

Abstract: Approaching the cultural dimension of companies is a recurrent, but also controversial and complex task. In its conceptualization, operationalisation and utilization in management, the organizational culture shows heterogeneous theoretical and empirical approaches, making it difficult for researchers to reach a consensus. Considering the company as a place of symbolic and identity creation that gives meaning and sense to the action of organization's members, the aim of the article is to revise from a critical standpoint the main theoretical and methodological approaches of organizational culture present in Organizational and Labour Studies. Moreover, this paper intends to identify the principal tensions and consensus existing around the concept and to weigh up his heuristic utility.

Key words: organizational culture, theoretical approaches, methodology.

\section{Introducción ${ }^{1}$}

La construcción de conceptos y categorías en las ciencias sociales es de por sí un asunto complejo y problemático. La transmutación de sentidos que algunos conceptos experimentan a lo largo del tiempo obliga a reflexionar y preguntarse sobre la utilización de los mismos y la significación que se les otorga en cada caso.

El término cultura es, quizás, uno de los más polisémicos, complejos y sobre-utilizados conceptos en las ciencias sociales. El amplio espectro de dimensiones, categorías e indicadores empíricos que incluye vuelven compleja la tarea de su delimitación teórica y aplicación en contextos específicos.
El abordaje de la dimensión cultural en las organizaciones $y$, específicamente, en las empresas es una temática relativamente reciente que despierta gran interés en los Estudios del Trabajo, los Estudios Organizacionales y los de Management. Más allá de sus divergencias, estos estudios presentan un interés común por relevar y analizar la vida simbólica de las organizaciones empresariales y sus miembros. Estos enfoques se han preocupado por dar cuenta de la manera en que las creencias culturales dan forma a la naturaleza y funcionamiento de las organizaciones así cómo por descifrar la información que la cultura nos brinda sobre la organización.

Tanto en su conceptualización, operacionalización y/o uso práctico (en la Administración de empresas), la

\footnotetext{
I Una versión preliminar de este artículo fue presentada en el VI Congreso de la Asociación Latinoamericana de Sociología del Trabajo (ALAST) realizado en Ciudad de México en abril de 2010.
} 
Cultura Organizacional (de ahora en más CO) presenta heterogéneos tratamientos que dificultan la construcción de consensos y la acumulación de conocimientos sobre la cuestión. Las definiciones, a menudo, suelen ser muy amplias o muy restringidas, incluyendo la combinación de otros conceptos tales como normas, valores, sentimientos, pensamientos, creencias, actitudes, etc. que asumen diferente énfasis según sea la perspectiva adoptada. La pluralidad de niveles y dimensiones de análisis hallada en los estudios sobre cultura y organización vuelve aún más confuso el contexto de discusión. Por ello resulta imprescindible explicitar la concepción que, no sólo del término cultura sino también del de organización, tenga cada uno de los enfoques considerados. Ambos conceptos se influyen mutuamente en el desarrollo analítico y de ellos dependerá la conclusión a la que se arribe en cada caso (Smircich, 1983).

En la literatura especializada sobre el tema podemos hallar diferentes posicionamientos que responden de manera particular a una serie de interrogantes. La cultura organizacional ¿constituye una variable más de la organización o es parte esencial, constitutiva, de su naturaleza?, ¿encuentra sustento en bases estructurales invariables o es fundamentalmente dinámica?, ¿constituye un mero reflejo de la cultura nacional o es un producto original de las interacciones existentes entre los miembros de la organización?, ¿asume características homogéneas o presenta divergencias y heterogeneidades en su interior?. Estas preguntas, creemos, esconden las tensiones fundamentales que atraviesan al concepto de C.O.

Adoptando una mirada regional, en América Latina los estudios organizacionales han tenido escaso desarrollo siendo eclipsados por los estudios de nivel macro, orientados al conocimiento de las tendencias productivas o sociales estructurales, y por la propensión a privilegiar en el análisis a los estados nacionales como la organización fundamental que explica el desarrollo en la región (Ibarra Colado, 2000). A pesar de ello, se han realizado importantes avances sobre la relación entre cultura y organización, especialmente en lo que respecta a los estudios del trabajo ${ }^{2}$. Así, la preocupación por «lo cultural» en la empresa constituye actualmente una línea de investigación relevante en el intento de explicar las trayectorias y prácticas organizacionales ${ }^{3}$.
Considerando a la empresa como un lugar de creación simbólica e identitaria, productora de conocimientos y dadora de significados y sentidos a la acción de sus miembros, el presente artículo tiene por objetivo revisar críticamente los principales abordajes teórico-metodológicos que, desde los Estudios de la Organización y el Trabajo, tuvo la cultura organizacional.

Por razones de espacio, no nos proponemos aquí hacer un recuento exhaustivo de las corrientes que abordan el fenómeno cultural en las organizaciones, sino situar los principales ejes de consenso y tensión existentes detrás del concepto. Ya existen importantes trabajos de síntesis que plantean diversas tipologías de los estudios sobre cultura y organización (Smircich, 1983; Martin, 1992; Hallet, 2003), que retomamos en la exposición. El trabajo se propone, entonces, identificar las divergencias y convergencias teóricas existentes en las diversas corrientes que abordan la $\mathrm{CO}$, así como las potencialidades que presenta el concepto en el estudio de las organizaciones y el trabajo.

Respecto al orden expositivo, en primer lugar, presentamos el contexto de surgimiento de los estudios sobre $\mathrm{CO}$ y los intereses teóricos y prácticos a los cuales respondían los primeros trabajos. En segundo lugar, planteamos los principales ejes que definen los posicionamientos teóricos existentes respecto al concepto para, a continuación, identificar los consensos subyacentes presentes en dichos posicionamientos.

En tercer lugar nos adentramos en la dimensión metodológica del concepto intentando relevar los principales nudos problemáticos existentes en la construcción de las mediaciones empíricas de la $\mathrm{CO}$. Finalmente se presentan una serie de reflexiones en torno al desarrollo realizado enfatizando las potencialidades del uso del concepto de CO.

\section{Contexto de surgimiento del concepto de cultura organizacional. Intereses teóricos y políticos en juego}

Si bien ya los primeros teóricos de la Sociología de las Organizaciones reconocían la existencia de la cultura y su influencia en el comportamiento de los actores organizacionales, refiriéndose a ella en forma indirecta en las reflexiones acerca del liderazgo o el

\footnotetext{
2 Para una revisión de la producción latinoamericana sobre esta cuestión consultar Montaño Hirose (2000).

3 Sin embargo, en las empresas de nuestra región los abordajes de la cultura organizacional o corporativa todavía aparecen más ligados a un discurso empresarial que a un proyecto de transformación real, ya que sigue predominando la cultura autoritaria y patriarcal más preocupada por el control que por el involucramiento de los trabajadores en la producción (Gallardo Velásquez, 200 I; Novick, 2000).
} 
clima organizacional ${ }^{4}$, es a partir de los años 80 que el término cultura organizacional adquiere entidad conceptual propia.

Desde la primera utilización del término en 1979, en un artículo de Pettigrew publicado en Administrative Science Quarterly, la CO parece haber asumido al interior de la teoría de las organizaciones una entidad teórica similar a la de conceptos clásicos como el de estructura, estrategia o control.

El surgimiento, a fines de los años 70, de la «temática cultural» en las organizaciones responde a un doble interés teórico y práctico. Por un lado, fue una respuesta teórica a la crisis de las explicaciones provenientes de los paradigmas racionalistas de la Administración (taylorismo). Por otro lado, respondió a motivaciones extra-teóricas que buscaban redefinir el modelo de gestión prevaleciente en las empresas, implementando nuevas formas de organización en contextos culturales diferentes. Nos referimos, específicamente, a los intentos de «importar» las formas de producción japonesas a las firmas de Estados Unidos y Europa.

Respecto al primer punto, el renovado interés por la cultura vino a legitimar la atención de los aspectos subjetivos e interpretativos existentes en la vida organizacional. Más allá de sus diferencias teóricas, las investigaciones que se interesan por la CO surgieron como respuestas críticas a las aproximaciones funcionalistas que enfatizaban la naturaleza racional y sistémica de las organizaciones.

El pionero trabajo de Silverman ( 197 I) constituyó una importante crítica al estructuralismo parsoniano al comprender a las acciones de los miembros de la organización como el resultado del sentido que los actores otorgan a la realidad social. Asumiendo un enfoque interpretativo, Silverman centró la mirada en las relaciones de significado construidas dentro de la organización para comprender su funcionamiento.

En relación al interés práctico, a partir de los trabajos de Peters y Waterman (1986) y Deal y Kennedy (1982) se acuña el término de cultura corporativa que viene a considerar el análisis de la cultura en la organización pero desde una perspectiva reduccionista. Se asume una visión funcionalista de los aspectos simbólicos de la organización y se los concibe como objetos manipulables que pueden influir sobre la productividad y la competitividad de la empresa. Las organizaciones consideradas «exitosas» serán, entonces, aquellas que tengan una cultura fuerte (Wilkins y Ouchi, 1983), una cultura compartida por todos sus miembros que se relaciona causalmente con los índices de compromiso y productividad laboral (Velasquez et al, 2010)

En este sentido, la preocupación por «la dimensión cultural» de las empresas debe situarse en el marco de los procesos de reorganización empresarial experimentados en las últimas décadas a nivel mundial. La concentración del capital y la expansión de las empresas trasnacionales hicieron que la multi-culturalidad comience a ser pensada como una variable más a tener en cuenta en la administración de las empresas. El interés de los empresarios en construir y establecer criterios corporativos homogéneos en los diferentes países donde opera la firma, fomentó la búsqueda de culturas corporativas propias que moldearan y controlaran el comportamiento de los miembros de la organización hacia el objetivo final de implementar la gestión flexible del trabajo y el aumento de la productividad.

La cultura en la organización fue concebida, entonces, como una variable susceptible de ser controlada, convirtiéndose «en una jaculatoria ideológica a través de la cual se instrumentan acciones concretas tendientes a modificar procesos y relaciones específicas que distan mucho de ser encasilladas como culturales» (Mendoza y Luna, 2000:27-28). Las funciones adjudicadas a la cultura en la organización, tales como creación de sentido de identidad, promoción del consenso y estabilidad del sistema y orientación del comportamiento de sus miembros (Peters y Waterman, 1982), pasaron a concebirse como instrumentos de gestión con un impacto directo sobre la eficiencia global de la organización. A menudo, el promovido compromiso del trabajador con su trabajo fue reducido a su compromiso con el aumento de la productividad empresarial. Es decir, que la propia idea de cultura corporativa esconde importantes implicancias políticas en su aplicación ${ }^{5}$.

Desde esta perspectiva adquiere un sentido central relevar y evaluar los valores que impulsan las accio-

\footnotetext{
${ }^{4}$ El concepto de clima organizacional hace referencia a «un set de propiedades mensurables del ambiente de trabajo percibido directa $\mathrm{o}$ indirectamente por la gente que vive o trabaja en dicho ambiente y que asume su influencia en sus motivaciones y comportamientos» (Litwin y Stringer (1968:I). Este concepto tiene una connotación evaluativa y a menudo se aprehende con medidas de satisfacción laboral. Proveniente de la Psicología Institucional, está más ligado a las motivaciones y satisfacciones individuales y puede ser subsumido bajo el término de cultura.

${ }^{5}$ Estos estudios sostienen que la satisfacción en el trabajo y el compromiso con la organización se correlacionan positivamente con la alta productividad de las empresas. Sin embargo todavía no hay evidencias concluyentes de que las prácticas de Recursos Humanos se relacionen positivamente con la performance de la empresa (Baldry, Hyman y Scholarios, 2005).
} 
nes de los actores dentro de la organización ya que se vuelve imprescindible conocer, primero, para movilizar saberes y capacidades productivas y orientar la fuerza de trabajo hacia actividades de mayor nivel de productividad, después. El modelo de gestión por valores, en este sentido, mantiene intacta la lógica de gestión capitalista de la mano de obra.

Asimismo, tanto en la bibliografía especializada sobre Management como en el propio discurso empresarial, la cultura organizacional comenzó a ser fuertemente asociada a los procesos innovativos llevados adelante por las firmas. En este sentido la cultura corporativa cobra, actualmente, un valor estratégico fundamental que deviene en una importante inversión por parte de las empresas en recursos económicos y humanos para su promoción y adopción. La calificación de los recursos humanos y la generación de un compromiso con los valores de la empresa surgen como dos pilares fundamentales en la generación de innovaciones (Morcillo, 2006), ya que dicho proceso implica, entre otros elementos, la movilización y transmisión de saberes individuales y colectivos, la transformación de los procesos de aprendizaje y la existencia de códigos comunicacionales compartidos.

Cabe señalar; sin embargo que no es objeto de este articulo analizar el carácter estratégico que asume actualmente la cultura organizacional en el desempeño de las empresas. A los fines del trabajo baste con destacar que la cultura corporativa ha adquirido en las ultimas dos décadas una relevancia creciente como promotora de la fidelización de los trabajadores a la organización y generadora de compromiso con la actividad productiva.

Sin embargo, esta concepción de la $\mathrm{CO}$ no ha sido la única perspectiva teórica, aunque sí la hegemónica, sobre la temática. En las últimas décadas han surgido otras interesantes aproximaciones de carácter más amplio que consolidaron el enfoque cultural de las organizaciones distanciándose claramente de los estudios de management.

\section{Las «divisorias de aguas» presentes en la conceptualización de la cultura organizacional}

En términos generales el concepto de cultura ha sido abordado en la Teoría de la Organización como una categoría «residual» o «cubo de basura» en la que se incluían aquellos aspectos que quedaban «por fuera» de las clásicas dimensiones del análisis organizacional (económica, productiva, de gestión y control, etc.).

De manera específica, la polisemia del concepto de CO nos presenta un amplio abanico de definiciones que va desde las enunciaciones holísticas y totalizadoras, que parten del supuesto de que «toda manifestación humana es cultural», hasta las más restringidas, que enfatizan sólo algún componente de la cultura. En este sentido, la $\mathrm{CO}$ se vuelve un concepto que parece prometer «todo o nada».

Por ello, partiendo del reconocimiento de la existencia de múltiples definiciones y abordajes metodológicos, se vuelve necesario identificar los principales ejes que determinan las diferencias entre las distintas corrientes analíticas. Como se verá a continuación, los criterios de clasificación pueden ser coexistentes, dependiendo de donde ubiquemos nuestro foco de análisis.

\section{I. Rango teórico}

Una primera gran diferenciación que puede establecerse entre las investigaciones sobre $\mathrm{CO}$ se refiere al rango teórico-analítico que se le otorga a la cultura. Así podemos diferenciar entre las que consideran a la cultura como un pilar esencial, constitutivo, de la organización y aquellas que entienden a la cultura como una variable de análisis más, como la económica o política. Es decir, la distinción radica en si se considera a la cultura como algo que «tiene»o algo que «es» la organización. La diferencia se establece entre una concepción de cultura como externa a los individuos (algo que «se tiene») o como «enraizada» en las interacciones de los sujetos o grupos que conforman la organización (algo que «se es») ${ }^{6}$.

Esta diferenciación es la que plantea Smircich (1983) en su modelo clasificatorio de los programas de investigación existentes en los años 80 sobre cultura organizacional, en el cual agrupa a los estudios comparativos de management y cultura corporativa en un primer grupo (que a su vez se diferenciará entre quienes consideren a la cultura como una variable externa o interna) y a aquellos que conciben a la cultura como una «metáfora», en tanto imagen ideal del

\footnotetext{
6 Por ejemplo Garmendia (1988) plantea la existencia de tres grandes perspectivas sobre la cultura de la empresa: el funcionalismo; que analizara a la CO como la cristalización del entorno, la perspectiva relativista, que la considerara como un diseño estratégico, y la perspectiva simbólica; que entiende a la cultura de la empresa como una imagen de sí misma.
} 
orden existente, en un segundo grupo. Estos últimos, a su vez, se diferenciarían entre sí según enfaticen los aspectos cognitivos, los aspectos simbólicos o los procesos subjetivos inconscientes existentes en la organización.

En el primero de los grupos (estudios del Management) las organizaciones son entendidas como organismos que existen en un ambiente que les presenta imperativos de comportamiento y al que deben adaptarse para sobrevivir. El interés fundamental radica en la búsqueda de «maneras o modos» predecibles de acción para controlar y mejorar la gestión. Hay una preocupación por la relación causal entre cultura y desempeño/rendimiento organizacional y productividad (Velasquez et al, 20 I0).

El segundo abordaje, que encuentra su raigambre teórica en la Antropología, reconoce a las organizaciones como construcciones sociales, manifestaciones de la acción y la conciencia humana, donde se construyen conocimientos, redes de significados y procesos psicológicos inconcientes (Smircich, 1983) que definen la propia naturaleza organizacional.

Entre las definiciones que podemos hallar dentro de este segundo grupo se hace referencia a la CO como aquellos patrones de significado que constituyen la base de manifestaciones observables (Martin, 1992), o como esquemas de interpretación y procesos de creación y negociación de significados (Sackmann, 199 I) que proveen un sentido al objetivo y la actividad de la organización a lo largo del tiempo (Clark y Soulsby, 1995).

Enfatizando su impronta práctica, Mills (1988), por ejemplo, define a la cultura como «esencialmente compuesta por un número de conocimientos (understandings) y esperanzas (expectations) que asisten a las personas en la construcción del sentido de la vida. (...) dichos conocimientos, que deben ser aprendidos, guían a las personas hacia el comportamiento adecuado, los ayudan a conocer cómo se hacen las cosas, que se espera de ellos, cómo alcanzar ciertos objetivos, etc.» (Ibid: 360) ${ }^{7}$.

Estos patrones de significado compartidos, se sostiene, son propios de cada organización ya que, según Hofstede, la $\mathrm{CO}$ es la «programación colectiva mental que hace distinguible a los miembros de una organización de otras» (Hofstede, 1991:262). La CO sería para la organización lo que la personalidad es para el individuo.

Planteada esta primera diferenciación, según la naturaleza que se adjudique a la cultura, podemos considerar una sub-división al interior del primer grupo que considera a la cultura en tanto variable. Así, diferenciamos los enfoques que buscan estudiar la cultura en el ámbito de la organización (variable interna) y aquellos que pretenden estudiar las características de la organización a partir de considerar la cultura nacional en la que esta se inserta (variable externa).

La cultura organizacional, en tanto variable, puede ser, entonces, objeto de dos miradas no siempre complementarias: 1) la interna, centrada en el proceso endógeno de creación de significados, y 2) la externa, centrada en la influencia del sistema de valores societales sobre la organización.

El primero de estos enfoques adopta una mirada intra-organizacional de la cultura $y$, desde ella, intenta comprender el comportamiento que asume la organización frente a eventos del entorno. Desde esta mirada se reconoce la naturaleza simbólico-significativa de la dinámica organizacional, pero se la concibe como una variable más capaz de explicar el comportamiento organizacional.

El segundo enfoque incumbe a aquellas investigaciones que analizan la influencia que tiene la cultura nacional sobre las estructuras organizacionales y/o los estilos de management (Crozier, 1963; Hofstede, 1980, D'Iribarne, 1989). La cultura es considerada, entonces, como una variable externa, que «introducen» los miembros de la organización.

Estos estudios entienden que las restricciones culturales de cada nación pesan sobre los individuos de la misma forma en que el vocabulario y las reglas de sintaxis intervienen en la construcción de un texto. La cultura nacional es entendida a modo de «gramática» que da forma y sentido a las prácticas de los actores organizacionales, en tanto constituye un «referencial de sentido» (D'Iribarne, 1989).

Las líneas de investigaciones en este enfoque se concentran, fundamentalmente, en la relación a nivel macro entre cultura nacional y estructura de las organizaciones ${ }^{8}$ y en el análisis micro de las similitudes y diferencias existentes en los modelos de gestión de

\footnotetext{
7 Todas las traducciones citadas y reproducidas pertenecen a la autora del articulo.

8 En esta línea D'Iribarne (1989), por ejemplo, pone en evidencia la continuidad histórico-cultural presente en la organización, enfatizando el papel de las estructuras sociales surgidas en épocas distantes que resurgen al nivel de la organización.
} 
empresas en diferentes contextos culturales. En términos generales, estos estudios concluyen que los valores de las organizaciones varían más de acuerdo a los criterios culturales y demográficos de sus miembros (tales como nacionalidad, edad o nivel de educación) que según las características propias de cada organización (Hofstede et al, 1990).

Más allá de los aportes realizados (sobre todo en el plano comparativo), dichos trabajos, a menudo, caen en el riesgo, por un lado, de equiparar cultura con nación, suponiendo la homogeneidad simbólica hacia el interior de las naciones y, por otro lado, de plantear una relación determinista entre los valores y rasgos culturales fundantes de una sociedad y el funcionamiento de las organizaciones (Crozier y Friedberg, 1990).

\subsection{Grado de diferenciación interna}

Desde la perspectiva intra-organizacional de la cultura, Meyerson y Martin (1987) reconocen la existencia de tres grandes corrientes que se diferencian entre sí según sea el grado de integración-diversificación que reconozcan al interior de la cultura organizacional. Estas serian las corrientes de I-integración, 2-diferenciación y 3-fragmentación.

La primera de ellas sostiene que las organizaciones comparten una única cultura, enfatizándose la consistencia, el orden y el consenso por encima de la diversidad y el conflicto. La cultura es usualmente definida como aquel «aglutinante» (g/ue) social y normativo que mantiene a la organización unida (Deal y Kennedy, 1982) y que expresa los valores y creencias que los miembros de la organización vienen a compartir por encima de sus diferencias. Estas visiones tienden a subestimar la existencia de subculturas o contraculturas que compiten por la asignación hegemónica de sentidos al interior de la organización.

Asimismo, sustentadas en buena parte de las premisas del estructural-funcionalismo, plantean que la integración a los valores de la organización genera compromiso (y mayor productividad) por parte de los trabajadores. De ahí la implicancia práctica que tiene esta perspectiva en la búsqueda de una «cultura fuerte» y controlada por la gestión empresarial (Wilkins y Ouchi, 1983).

Desde la perspectiva de la diferenciación se acepta la existencia de sub-culturas definidas por factores tales como el género, la jerarquía, el origen étnico o la condición migratoria que, teniendo valores, percep- ciones y actitudes diferentes, conviven, se enfrenta y negocian entre sí al interior de la organización. De allí que la organización sea percibida como un «orden simbólico negociado» (Mills, 1988).

Finalmente la perspectiva de la fragmentación sostiene que la cultura es esencialmente ambigua y dinámica. Las percepciones y valores sustentados cambian según las personas que integran la organización y las situaciones a las cuales ella se enfrenta. Por ello, el consenso que supone la existencia de una $\mathrm{CO}$ es reconstruido de manera permanente y no siempre alcanza una cristalización satisfactoria. Esta posición podría legar al punto de negar la existencia de una $\mathrm{CO}$ en tanto unidad analítica.

El propio Martin (1992) afirma que estos tres enfoques deben ser considerados como complementarios más que como excluyentes, ya que iluminan aspectos diferentes de la cultura y son útiles al momento de reconstruir los espacios de integración, diferenciación y fragmentación existentes al interior de toda organización. Una misma cultura organizacional permite albergar en sí misma lo homogéneo, lo diverso y aún lo contradictorio.

\subsection{Relación con el entorno}

Si bien la relación de la organización con el entorno constituye ya una línea de investigación clásica en la Teoría de las Organizaciones, es una dimensión que resurge al revisitar los desarrollos teóricos sobre $\mathrm{CO}$. El grado de dependencia o autonomía de la $\mathrm{CO}$ respecto al contexto en el que se inserta es otro de los ejes que distancian entre sí a las investigaciones desarrolladas. Así, hallamos enfoques que tienden a enfatizar la dependencia del entorno, en tanto se lo considera como la principal variable explicativa de las características que presenta la $\mathrm{CO}$, y otros que refuerzan la visión de la $\mathrm{CO}$ como un orden negociado resultado de las interacciones entre los actores, que son quienes perciben y «mediatizan» de modo original las influencias del entorno.

Sumado a la corriente de los estudios transculturales, que se incluyen claramente en la primera de las perspectivas señaladas, existen nuevos enfoques de corte funcionalista que plantean que las organizaciones no tienen culturas tan «propias» o características sino que son, en gran medida, una proyección cultural de sus contextos. Se habla, entonces, de culturas industriales, según sector de actividad, y de subculturas ocupacionales o profesionales, que tendrían más incidencia sobre el comportamiento de los 
miembros de la organización que los rasgos asociados a la unicidad de la CO.

También aquí pueden incluirse las perspectivas funcionalistas que consideran a la cultura como variable. Según Schein (1999) la cultura «corporativa» es «un modelo de presunciones básicas -inventadas o descubiertas por una organización para resolver sus problemas- que ejercen influencia como para ser consideradas válidas y, en consecuencia, ser enseñadas a los nuevos miembros como el modo correcto de percibir, pensar y sentir tales problemas». Cuando esta solución sirve de manera repetitiva a la resolución de los problemas, se va asentando de manera que los comportamientos se convierten en premisas básicas de actuación.

Para esta perspectiva los cimientos de una cultura organizacional se estructuran cuando la gente debe afrontar simultáneamente un problema y concebir juntas una solución. La CO se explicaría, entonces, como resultado de la relación de la organización con su entorno en tanto acumulación de experiencias y conocimientos ganada en el proceso de adaptación de la organización al contexto.

Asimismo, retomando algunos supuestos de la teoría de la Contingencia, se señala que son las condiciones iniciales de «ajuste o desajuste» de la cultura con su entorno las que explican la eficiencia de la organización (Kotter y Heskett, 1992) y que es posible influir en la evolución satisfactoria de las empresas mediante la promoción y gestión de determinados valores (Vallejo Martos, 2008).

Pero al mismo tiempo la CO influye en la percepción que se tenga de dicho entorno creando un doble condicionamiento. Desde esta perspectiva Krieger señala que «la cultura ayuda a interpretar la naturaleza y significado de las relaciones organización-entorno. (...) El conocimiento y las relaciones con el entorno son extensiones de la cultura ya que llegamos a saber y comprender nuestro entorno a través de sistemas de creencias que guían nuestras interpretaciones y acciones» (Krieger, 2000:357).

Intentando realizar una síntesis entre las clasificaciones planteadas por Smircich (1983) y Meyerson y Martin (1987), Hallett (2003) plantea que las diferentes aproximaciones de la $\mathrm{CO}$ se apoyan en tradiciones sociológicas disímiles. Una de ellas, a la cual el autor adhiere será el considerado enfoque «práctico» o «del poder simbólico», que trata de articular las contribuciones teóricas de la práctica (Bourdieu) con las de la interacción (Goffman), considerando a la $\mathrm{CO}$ como un orden negociado donde se pone en juego el poder simbólico de los actores de definir la situación. Los significados no están situados al nivel de los procesos individuales internos ni en los confines del contexto cultural, sino que son construidos como producto de las interacciones sociales y de los procesos institucionales (Lee, 2000, Silverman, 197I)

De manera específica Hallett (2003) define a la cultura organizacional como «un orden negociado que emerge de las interacciones entre los actores, un orden influenciado particularmente por gente con poder simbólico, el poder de definir la situación en donde las interacciones tienen lugar» (2003:|30). La cultura organizacional se explica a partir de las acciones de los actores, que redefinen significados permanentemente aunque, en apariencia, la organización no cambie. Las prácticas e interacciones de los actores tienen un rol constitutivo en la construcción de las estructuras organizacionales y, claramente, de la CO.

Esta perspectiva interaccionista también es compartida por Crozier y Friedberg (1990) para quienes la cultura «es instrumento y es capacidad que adquieren, utilizan y transforman los individuos al tiempo que construyen y viven sus relaciones e intercambios con los otros (...)» (|990: |72). Desde este enfoque las influencias del entorno se encuentran siempre medidas por los juegos de poder existentes en la organización. En este sentido, según señalan Mendoza y Luna (2000), se trata de «adoptar una visión que reconozca la particularidad de los distintos arreglos que resultan de las combinaciones de fuerzas e informaciones del exterior que llegan a los grupos humanos y que, sin embargo, al ser incorporados a sus esquemas de interpretación los dotan de un sentido nuevo, dando a sus significados, pretendidamente originales, una suerte de resignificación (...). En esta óptica las organizaciones serían percibidas como arenas cruzadas por infinidad de influencias y procesos a las que no se les puede adjudicar mecánicamente la capacidad de percibir de manera idéntica procesos que sólo son semejantes al nivel de la definición formal» (2000:38).

Ahora bien, según sea el posicionamiento tomado respecto a los ejes de discusión planteados más arriba, cada enfoque adoptará objetivos de investigación y niveles de análisis diferentes.

El objetivo presente detrás de cada investigación, aunque no siempre este sea tan claro de determinar, puede variar entre la intención teórico-académica de conocer y comprender los procesos organizacionales y el propósito práctico, prospectivo, de «medir»la 
CO para gestionarla y administrarla de modo de promover el cambio cultural para hacerlo «coherente» con la estrategia empresarial (De Witte y van Muijen, 1999) 9 .

Por otro lado, el posicionamiento teórico también determinará el nivel de análisis privilegiado. Así podemos encontrar investigaciones que involucran desde estudios comparativos trans-culturales, análisis de culturas nacionales, de culturas profesionales y de sectores productivos hasta abordajes de culturas organizacionales (corporativas) específicas.

\section{Consensos teóricos de mínima}

Según lo expuesto hasta aquí, la multiplicidad y heterogeneidad parecen ser los rasgos definitorios de la teorización sobre la CO. Sin embargo, en dicha diversidad pueden identificarse algunos puntos de consenso que sirven de apoyo para la construcción de una corriente analítica de cierta densidad teórica. Se- ñalamos, a continuación, los que, a nuestro entender, pueden ser los acuerdos más relevantes para tal fin.

1) Todos los enfoques descriptos conciben a la organización, en mayor o menor medida, como un lugar de creación simbólica e identitaria, productora de conocimientos y dadora de significados y sentidos a la acción de sus miembros. Según el rango teórico que se le otorgue a la cultura, para algunos esta será una característica secundaria, que no define la «naturaleza» de la organización, mientras que para otros será una condición propia de su existencia; pero, en todos los casos se reconoce la existencia de relaciones simbólicas que influyen en la formación de las organizaciones y las prácticas de los sujetos (Tayeb, 1994).

En este sentido, se acepta que la cultura es una variable explicativa central para analizar la permanencia o el cambio de los esquemas interpretativos compartidos que hacen posible la organización a lo largo del tiempo.

Figura I

Ejes conceptuales de diferenciación

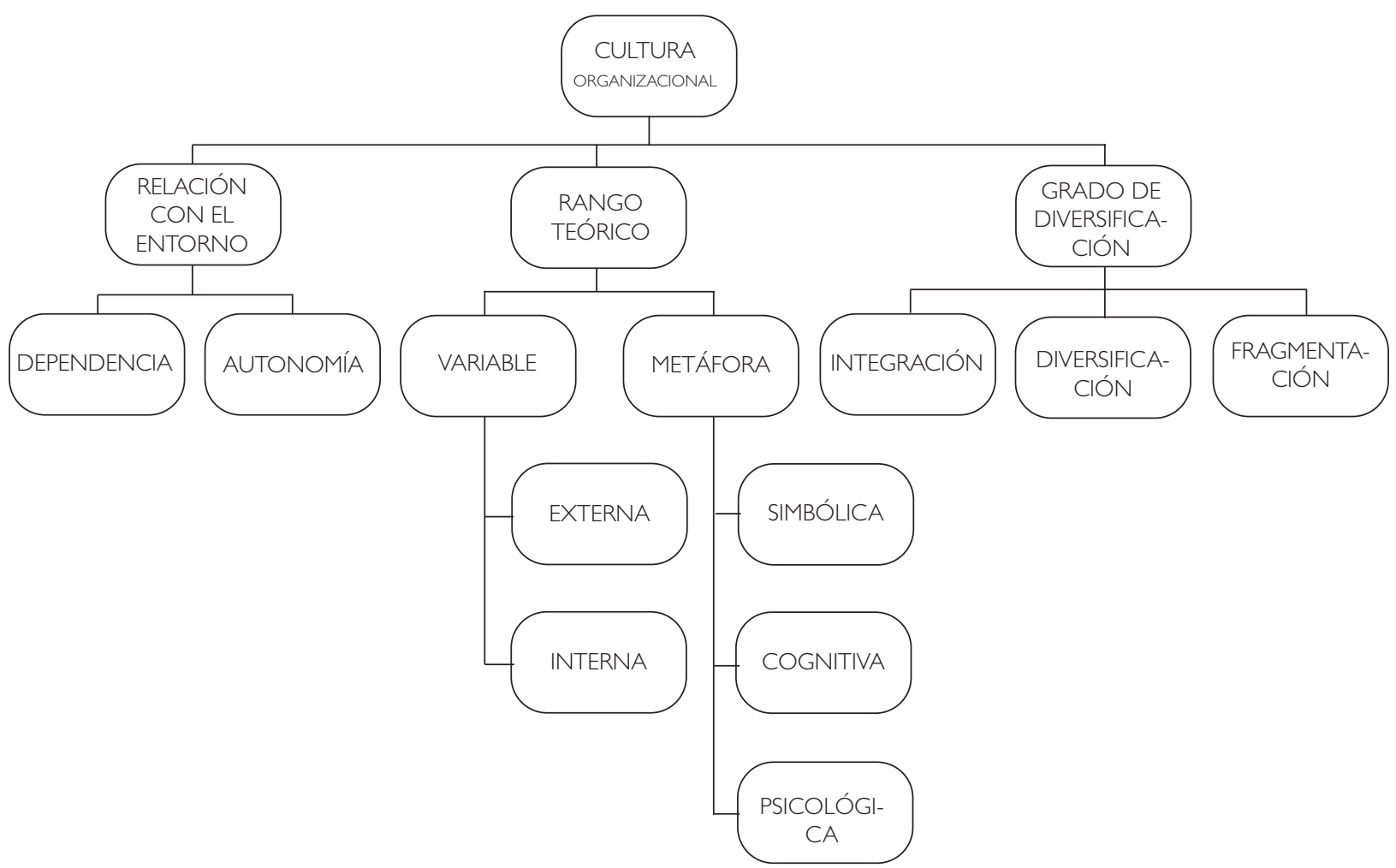

Fuente: Elaboración propia.

\footnotetext{
9 En las ultimas dos décadas las empresas han invertido importante cantidad de recursos humanos y económicos en la gestión de la cultura corporativa. En este sentido la temática cultural cobra una relevancia creciente en términos de inversión empresarial.
} 
2) Más allá de los diferentes énfasis, se consensúa que la cultura afecta el comportamiento de los miembros de la organización, interviniendo en los cursos de acción seguidos por los sujetos. La cultura, se reconoce, otorga fundamento a la acción y actúa a modo de «guía» o «mapa de ruta» de los actores organizacionales. Como señala Sackmann ( I99|), la CO se refiere al conjunto de «creencias sostenidas en común por los miembros de un grupo (...) que los actores utilizan como un mapa de ruta para funcionar en un contexto organizacional particular»» (1991:303). De allí la preocupación teórico-práctica, especialmente desde la perspectiva de los estudios del management, por describirla y comprenderla para, luego, modificarla y «adaptarla» a los nuevos requerimientos «del ambiente» (Ranieri, 1998).

3) Otro de los acuerdos básicos al que llegan los distintos autores es que la cultura tiende a preservar, en mayor o menor medida, sus rasgos centrales a lo largo del tiempo. La relativa estabilidad de la CO, aún en contextos de profundos cambios en el entorno económico, productivo y/o institucional, es un rasgo subrayado en la bibliografía especializada. Las estructuras simbólicas de la organización, según lo demuestran numerosas investigaciones, no se modifican de manera directa e instantánea al transformarse el entorno en el que se insertan (Kostera y Wicha, 1996; Clark y Soulsby, 1995). Dichas estructuras tienden a persistir a pesar de los cambios «externos».

Al reconocerse que la cultura tiene que ver con el aprendizaje acumulado por los miembros de la organización a lo largo de su historia, se acepta que la CO tenga otra «velocidad» de transformación que la vuelve difícilmente modificable en el corto plazo.

4) A pesar de los diferentes énfasis puestos según el enfoque, en términos generales, se reconoce el carácter dinámico y heterogéneo de la CO. Pocos autores cuestionarían que la cultura es esencialmente dinámica y que está en construcción y redefinición permanente, producto de la relación con el entorno y de las interacciones ocurridas entre los actores al interior de la organización. El reconocimiento de la CO como un producto socio-histórico implica la aceptación de su rasgo esencialmente dinámico.

Asimismo, aunque todavía existen perspectivas teóricas que enfatizan el carácter integrador de la cultura, en la bibliografía se acepta cada vez más la existencia de sub-culturas y grupos diferenciados al interior de la organización. En este sentido, se admite que en las organizaciones «existen muchos sistemas de valores diferentes y competitivos que crean un mosaico organizacional entre diferentes grupos, más que culturas corporativas uniformes» (Krieger 2001:337).

\section{La traducción empírica del concepto. Abordajes metodológicos múltiples}

Otro de los ejes que genera debate entre la bibliografía especializada sobre $\mathrm{CO}$ se refiere a la aprehensión empírica del concepto. De acuerdo a cual sea la perspectiva teórica adoptada (cuya heterogeneidad planteamos en los apartados anteriores) se derivarán las elecciones metodológicas respecto al nivel de análisis, las técnicas utilizadas, las fuentes privilegiadas y las estrategias analíticas desarrolladas en la investigación. El posicionamiento teórico definirá la operacionalización que se haga del concepto y las relaciones lógicas que se establezcan entre los indicadores.

El primer punto problemático, y ya lugar común en las ciencias sociales, se establece en torno a la utilización de métodos de análisis cuantitativos y cualitativos. Dicha división atraviesa los estudios sobre CO despertando prejuicios de uno y otro lado.

Según Sackmann (199|) existen dos grandes perspectivas metodológicas en el estudio de la cultura: una perspectiva «outsider», deductiva, más cercana a los métodos cuantitativos, que concibe a la cultura como una variable objeto de medición y control, y otra «insider», inductiva, preocupada por interpretar a la organización, a partir de la utilización de técnicas cualitativas, en tanto expresión situada de la cultura. Entre estas dos perspectivas puede construirse un continum de técnicas de investigación diversas, tanto de corte cuantitativo como cualitativo, que incluye desde la aplicación de cuestionarios (estándares o diseñados para la propia organización), análisis de decisión multicriterios, realización de entrevistas y análisis documental hasta discusiones en grupos focalizados y desarrollo de observaciones participantes. Como bien señala Sackmann (199|), adoptando una mirada metodológica integradora, la CO no es asunto exclusivo de uno ni otro método sino que la utilización de técnicas cuantitativas o cualitativas dependerá de los objetivos planteados en la investigación.

Un segundo problema metodológico recurrente en la temática es el de la definición y correspondencia entre la unidad de análisis teórica y las unidades empíricas utilizadas para recolectar la información. Las manifestaciones de la $\mathrm{CO}$ se observan y miden, fundamentalmente, a partir del comportamiento, las per- 
cepciones e ideas de los individuos. La objeción que se señala a varios estudios se refiere a la inferencia que realizan de los resultados individuales (obtenidos a partir de las «mentes» de los sujetos) hacia la unidad analítica de la organización, lo cual plantea un problema de agregación metodológica (Hofstede, 1998).

Este punto también esta relacionado con la tendencia, presente en algunas investigaciones, a atribuir características humanas a la unidad analítica de la «organización». A menudo la $\mathrm{CO}$ es analizada desde perspectivas que utilizan metáforas psicológicas para dar cuenta de ella. Así, se hace referencia al «carácter» (Selznick, 1957) o la «personalidad» (Morgan, 1986) de la organización atribuyéndole a esta comportamientos humanos y operando, de esta manera, un proceso de reificación.

Un tercer punto se refiere al problema concreto de la operacionalización ya que, al momento de revisar las categorías empíricas utilizadas para aprehender la CO las opciones son múltiples, volviéndose a presentar el mismo problema de la polisemia conceptual. A menudo, el uso indiferenciado de términos tan disímiles como el de actitudes, valores, ideas, normas, percepciones o expectativas como indicadores de la CO enturbia la comprensión de la construcción y operacionalización que se realiza del concepto (Hofstede, 1998).

Mientras que Hofstede (1990), por ejemplo, identifica a los símbolos, los héroes, los rituales y los valores como categorías centrales en las que agrupar las manifestaciones culturales, el modelo metodológico propuesto por Schein (1982) se compone de tres niveles, conformados por los artefactos culturales, los valores y las presunciones básicas compartidas, que abarca desde los componentes más visible hasta los tácitos e intangibles de la CO.

En la revisión bibliográfica pueden encontrarse distintos niveles de «profundidad» en los indicadores construidos, que involucran una multiplicidad de manifestaciones culturales de carácter material y simbólico. Como bien señala Sackmann (199|), las manifestaciones materiales se encuentran en un «nivel superficial» mientras que los componentes estructurales de la cultura a menudo no se manifiestan directamente y requieren de una compleja metodología para su aprehensión.

En este sentido, el abordaje de las expresiones culturales materiales no es suficiente, ya que «un entendimiento de la cultura en un marco organizacional dado requiere la comprensión de los aspectos conceptua- les (ideational) - los procesos de creación de sentido, las construcciones cognitivas o el conocimiento cultural que existe en una organización particular y es usado para atribuir significado al comportamiento observable y a los artefactos corporativos» (Sackmann, 1991:298). Sin embargo, la comprensión de este aspecto nunca puede estar divorciado de los comportamientos observables ya que, como recuerda Martin (1992), «es engañoso representar a la cultura en las organizaciones como mundo secretos de ideas y valores desconectados de las prácticas») (Ibid: 42).

Más allá de las preferencias metodológicas las tendencias generales concuerdan en promover la integración de métodos en la tarea de reconstruir la trama cultural específica de cada organización, recomendando para ello la utilización de metodologías de tipo inductivas.

Los señalamientos aquí realizados vuelven a ubicar a la metodología en el campo de decisión del propio investigador ya que serán las medidas que este tome y explicite las que determinarán la «solución» a los problemas señalados. En qué grado la cultura se subordina a la interpretación que los actores organizacionales hacen de ella, qué mediaciones empíricas no «sujetas» a los individuos podemos construir para abordar la $\mathrm{CO}$ o qué tipo de indicadores debemos priorizar en el análisis son interrogantes que cada investigador debe plantearse y responderse de manera particular dada la implicancia que estas decisiones tienen sobre los resultados alcanzados.

\section{Reflexiones finales}

Como hemos señalado en este brevísimo recuento de la bibliografía, la noción subyacente que cada teoría tiene de la organización y la cultura es definitoria de las conclusiones a las que se arribe en el análisis. Los resultados empíricos son siempre construidos e iluminados por las premisas teóricas que asuma el investigador.

Ahora bien, conociendo las disputas conceptuales y metodológicas que este despierta, podríamos preguntarnos cuáles son las supuestas ventajas de seguir utilizando un concepto tan controvertido y polisémico como el de CO. Más allá de las diferencias teórico-metodológicas señaladas, sostenemos que la multiplicidad de perspectivas parece enriquecer más que enturbiar el campo analítico de la cultura organizacional.

Desde nuestra perspectiva creemos que el concepto guarda importantes potencialidades para el estu- 
dio de las organizaciones y el trabajo en ellas desarrollado, en tanto ilumina una dimensión poco explorada en estas, que se refiere a los elementos simbólicos que la constituyen y que influyen en el comportamiento de los actores.

Entre los avances más relevantes hechos desde la perspectiva de la $\mathrm{CO}$ se destaca el planteo teórico de una nueva mirada (interpretativa) de la organización que reconoce la relevancia que tienen los procesos de comprensión y significación en las acciones de los actores que crean y mantienen el sentido de la organización (Mills, 1988).

Siguiendo a Tayeb (1994) podemos señalar que la perspectiva cultural en los estudios organizacionales legitimó el reconocimiento de que las actitudes y valores culturales son diferentes entre una sociedad y otra, que los grupos culturales se comportan de manera diferente frente a iguales circunstancias y que la cultura juega un rol importante en la formación de las organizaciones y las prácticas de los sujetos.

En este sentido, creemos, que el verdadero aporte del concepto de cultura organizacional es convocar la atención sobre las dimensiones intangibles, difícilmente aprehensibles, de las organizaciones. De esta manera, la potencialidad del término, quizás, no se sitúe en generar respuestas a las tradicionales preguntas de la Teoría de la Organización sino en dejar planteados nuevos interrogantes que ahonden en el conocimiento de las organizaciones y el trabajo.

Creemos que la cultura tiene aún mucho que decir (y explicar) sobre el comportamiento productivo y organizacional de las empresas. Si bien este puede no ser el más importante ni el único de los aspectos de la organización, es fundamental para comprender los procesos de construcción organizacional.

Para ello, sin embargo, es necesario apartarse de la perspectiva hegemónica y reduccionista de los estudios sobre cultura corporativa que comprenden a los valores organizacionales únicamente como elementos estratégicos internos que permiten aumentar la productividad. Asumir una concepción amplia y, fundamentalmente, constructivista de la $\mathrm{CO}$, donde el universo simbólico de la organización se explique a partir de las interacciones desarrolladas históricamente entre los actores, es una de las maneras de enriquecer el campo de discusión y de generar nuevos interrogantes. Concebir a la cultura como un orden negociado, «como una arena de disputa por la asignación de significaciones referidas a procesos concretos de interacción social que implican hombres de carne y hueso, con intereses siempre referidos a algo 0 a alguien», es regresar el debate a la tierra (Mendoza y Luna, 2000:42).

Adoptar una perspectiva que se adentre en el estudio de las organizaciones dando cuenta de «las sutilezas que involucran los procesos figurativos (y) los arreglos particulares simbólico-significativos que se producen en organizaciones concretas» (Mendoza y Luna, 2000:30) es el primer paso en el intento de construir teorías de alcance medio que puedan escapar a los riesgos tanto de la generalización rápida de resultados como de la sujeción del análisis a unidades empíricas específicas. El desarrollo de estudios de caso, concretamente situados, en organizaciones teóricamente representativas puede representar un importante aporte en este sentido.

\section{Bibliografía}

BALDRY, C. HYMAN, J.y SCHOLARIOS, D. (2005), «Knowledge workers, commitment and occupational community», Internacional Labour Process Conference 2005. University of Strathclyde, Glasgow.

CLARK E. y SOULSBY A. (1995), «Transforming Former State Enterprises in the Czech Republic», Organization Studies, 1995; 16;215-242

CROZIER, M. y FRIEDBERG, E. (1990), El actor y el sistema. Las restricciones de la acción colectiva, Alianza Editorial Mexicana, México.

CROZIER, MICHEL (1963, 1974), El fenómeno burocrático, Amorrortu, Buenos Aires.

DE WITTE, K. y VAN MUIJEN, J. (1999), «Organizational Culture: critical questions for researchers and practitioners», en European Journal of Work and Organizational Psychology, 1999, 8 (4), 583-595.

DEAL, T. y KENNEDY, A. (1982), Corporate cultures: The rites and rituals of corporate life, Reading MA: AddisonWesley.

D'LRIBARNE, PHILIPPE (1989), La Logique de I'honneur. Gestion des entreprises et traditions nationales. Éditions du Seuil. Paris, Francia.

GALLARDO VELÁSQUEZ, A. (200 I), «El debate de paradigmas en torno a la cultura organizacional» en Administración y Organizaciones, Julio 2001.

GARMENDIA MARTINEZ, JOSE (1988), La cultura de la empresa, una aproximación teórica y practica», en Revista Española de Investigaciones Sociológicas, Num. 4I, pp. 7-23.

GEERTZ, CLIFFORD (1973), Interpretation of Cultures, New York ; Basic Books. 
GRINT, K. (1995), Management : A Sociological Introduction, Cambridge, Polity Press.

HALLETT,TIM (2003), «Symbolic Power and Organizational Culture», en Sociological Theory, 21 :2 June 2003, Washington

HOFSTEDE, E., NEUIJEN, B., DAVAL, D. y SANDERS, G. (1990), «Measuring Organizational Cultures: a qualitative and quantitative study across twenty cases»), en Administrative Science Quarterly, 35 (1990), 286-316.

HOFSTEDE, G. (1998), «Attitudes, Values and Organizational Culture: Disentangling the Concepts», Organization Studies 1998; 19; 477-492.

HOFSTEDE, GEERT (1980), Culture's consequences, Sage, London.

IBARRA COLADO, EDUARDO (2000), «iEstudios organizacionales en América Latina? Transitando del centro hacia las orillas», en De la Garza Toledo, E. (coord.) (2006), Teorías sociales y estudios del trabajo: nuevos enfoques, Anthropos, México.

IBARRA COLADO, EDUARDO (2000), «Teoría de las organizaciones, un mapa conceptual en disputa», en De la Garza, E. (comp.) (2000) Tratado Latinoamericano de Sociología del Trabajo, Fondo de Cultura Económica, México.

KOSTERA, M. y WICHA, M. ( 1996), «Organizing The 'Divided Self' of Polish State-owned Enterprises:The Culture of Organizing», Organization Studies 1996; 17; 83- 105

KOTTER, J.y HESKETT, J. ( 1992), Corporate Culture and Performance, Nueva York, The Free Press.

KRIEGER, MARIO (200 I), Sociología de las organizaciones, Prentice Hall / Pearson Education, Bs. As.

LEE, O. (2000), «The constitution of Meaning : On the Practical Conditions of Social Understanding », Current Perspectives in Sociological Theory, 20 :27-64.

LITWIN, G. y STRINGER, R.(1968), Motivation and organizational climate. Boston: Graduate School of Business Administration, Harvard University.

MARTIN et al (1983), «The Uniqueness Paradox in Organizational Stories», en Administrative Science Quarterly, $28: 438-453$.

MARTIN, JOANNE (1992), Cultures in organizations: Three perspectives. Nueva York: Oxford University Press.

MENDOZA, R. y LUNA, A. (2000), «La cultura en las organizaciones. Acerca de la utilidad de las perspectivas de corto alcance», en Administración y Organizaciones, Julio 2000

MEYERSON, D. y MARTIN, J. (1987), « Cultural change : An integration of three different views $\gg$, Journal of Management Studies, 24(6) : 623-47.
MILLS ALBERT ( 1988), «Organization, Gender and Culture», Organization Studies 1988; 9; 351-369

MONTAÑO HIROSE, LUIS (2000), «La dimensión cultural de la organización», en De la Garza, E. (comp.) (2000) Tratado Latinoamericano de Sociología del Trabajo, Edit. Fondo de Cultura Económica, México.

MORCILLO, Patricio (2006) Innovación y cultural empresarial. La conexión perfecta. Thomson Paraninfo, Madrid.

NOVICK, MARTA (2000), «La transformación de la organización delTrabajo», en De la Garza, E. (comp.) (2000) Tratado Latinoamericano de Sociología del Trabajo, Fondo de Cultura Económica, México.

PETERS, T. y WATERMAN, R. (1 982), In search ofexcellence: lessons from America's best-run companies. New York: Harper \& Row.

PETTIGREW, A. ( 1 979), « On Studing Organizational Cultures», Administrative Science Quarterly 24, 570-81.

RAINERI, ANDRÉS (1998), «Creencias y cambio organizacional en los sectores público y privado», en Estudios Públicos, 70, Otoño 1998.

Rituals of corporate life, Reading MA: Addison-Wesley.

SACKMANN, S. (1991), «Uncovering Culture in Organizations», en Journal of Applied Behavioral Science |99|; $27 ; 295-317$

SCHEIN, E. (1982), La cultura empresarial y el liderazgo, Prentice Hall, Madrid.

SCHEIN, EDGARD (199|). «What is culture?» in P. Frost, L. Moore, M. Louis, C. Lundberg and J. Martin, Reframing Organizational Culture. London: Sage.

SILVERMAN, DAVID (1970), Teoría de las organizaciones, Nueva Visión, Buenos Aires.

SMIRCICH, LINDA ( 1983), «Concepts of Culture and Organizational Analysis», en Administrative Science Quarterly, 28 (1983) : 339-358.

TAYEB MONIR, ( 1994), «Organizations and National Culture: Methodology Considered», Organization Studies 1994; 15; 429-445

VALLEJO MARTOS, MANUEL C. (2008), «CORAJE: la respuesta cultural a los problemas evolutivos de la empresa familiar», en Dirección y Organización, Núm. 36, Octubre 2008, 51-60.

VELASQUEZ,Y., NÚÑEZ, M. y RODRÍGUEZ MONROY, C. (20 I0), «Aplicación de la técnica AHP para evaluar el efecto de los valores organizacionales en la productividad», en Dirección y Organización, Núm. 4I, Julio 2010,58-67.

WILKINS A. L.y OUCHIW.G. ( 1 983) «Efficient culture: Exploring the relationship between culture and organization performance». Administrative Science Quarterly $28,468-481$ 